\title{
Effects of Virtual Reality Head-mounted Displays on Oculomotor Functions
}

\author{
Mosaad Alhassan", Fatimah Alhamad, Kholoud Bokhary, Ali Almustanyir \\ Department of Optometry, College of Applied Medical Sciences, King Saud University, Riyadh, Kingdom of Saudi Arabia \\ Email address: \\ malhassan@ksu.edu.sa (M. Alhassan), fatimah.h.ksu@gmail.com (F.h Alhamad),kbokhary@ksu.edu.sa (K. Bokhary), \\ aalmustanyir@ksu.edu.sa (A. Almustanyir) \\ ${ }^{*}$ Corresponding author
}

\section{To cite this article:}

Mosaad Alhassan, Fatimah Alhamad, Kholoud Bokhary, Ali Almustanyir. Effects of Virtual Reality Head-mounted Displays on Oculomotor Functions. International Journal of Ophthalmology \& Visual Science. Vol. 6, No. 1, 2021, pp. 10-16. doi: 10.11648/j.ijovs.20210601.12

Received: January 23, 2021; Accepted: February 1, 2021; Published: February 9, 2021

\begin{abstract}
Purpose: Three-dimensional (3D) video games played using virtual reality head-mounted displays (VR-HMDs) are becoming increasingly popular. However, the use of this technology may lead to visual symptoms and discomfort by disrupting the normal linkage between the accommodation and vergence systems. This study aimed to investigate the effect of playing 3D video games using VR-HMDs on different oculomotor functions (i.e., accommodation and/or vergence system parameters) and to quantify symptoms associated with playing games using VR-HMD technology. Methods: Twenty -six male and female young adults (age range 19-27 years) with normal binocular vision completed this study. Different clinical accommodative and vergence measurements were collected before and after playing 3D video games using a VR-HMD system for 45 minutes. In addition, visual- and non-visual-related symptoms were measured before and after the video game sessions using the standardized Simulator Sickness Questionnaire. Results: The majority of accommodative parameters-including negative relative accommodation, accommodative accuracy, and monocular and binocular accommodative facilities-were significantly affected after playing 3D video games. With respect to vergence system measurements, the horizontal negative fusional vergence range at near and vergence facility test outcomes were affected significantly after the 3D video game sessions. Significant increments in different types of symptoms (i.e., visual and nonvisual) were also observed after playing 3D video games using the VR-HMD system. Conclusions: Playing 3D video games using VR-HMD systems can lead to a deterioration of certain oculomotor functions (i.e., accommodative and vergence systems). Players can be expected to experience eyestrain and discomfort after just 45 minutes of playing.
\end{abstract}

Keywords: Virtual Reality, Video Games, Cybersickness, Oculomotor, Accommodation, Vergence, Binocular Vision, Simulator Sickness Questionnaire

\section{Introduction}

State-of-the-art virtual reality (VR) systems allow users to view stereoscopic three-dimensional (3D) video games or movies using binocular head-mounted display (HMD) devices. This technology is quickly gaining interest among young adult individuals looking for enjoyable activities to participate in [1]. However, though VR-HMDs have improved the quality of the video-game-playing experience over that of traditional 2D display systems, some important limitations persist. Watching a 3D film or playing a $3 \mathrm{D}$ video game for a long time can cause a significant increase in visual discomfort, eyestrain, and other nonvisual symptoms
$[2,3]$. Cybersickness" is a term used to describe the symptoms such as eye discomfort, headaches, and nausea that are experienced after watching a 3D film or playing a $3 \mathrm{D}$ video game [4].

Humans normally have a pair of eyes located in two different lateral positions in the head, which results in a slight shift of the viewing angle of the perceived images between the two eyes; this phenomenon is known as "retinal disparity" or "horizontal disparity" [5]. During natural viewing conditions, the accommodation and vergence systems in the eyes normally work together to produce a clear and fused 
single image, even when processing data from variable viewing distances over long periods of time, which results in one persistent stereoscopic image. Thus, when a human's eyes accommodate (i.e., focus on a near object), the eyes converge toward that object. Similarly, when the accommodation is relaxed (i.e., focusing on a far object), the eyes diverge at the same time. However, when a person watches a 3D movie-using, for example, VR-HMD technology - the image either floats toward the eyes or backward from the screen plane. If the former is true, the eyes converge to keep the perceived image fused into a single image. At the same time, accommodation will be stimulated to focus on the presented image as it moves closer. However, this is an issue because the image is not actually moving toward the eyes; instead, it remains located in the plane of the screen, so the increase in accommodation may make the image appear blurry, causing the accommodation to relax and the eyes to diverge. This triggers a disruption in the normal linkage between the accommodation and vergence systems known as "accommodation-vergence conflict." This extra work performed over a long period leads more rapidly to visual discomfort and fatigue [6].

There are also other factors that may contribute to the occurrence of visual and nonvisual symptoms after watching a $3 \mathrm{D}$ movie, including the crosstalk phenomenon, which is characterized by an incomplete dissociation between the respective images seen by the right and left eyes. An excessive amount of retinal disparity between the two images places a large demand on the vergence system to fuse the two images. Image distortion is another factor related to the technical problems of the device [7].

Symptoms that are commonly exhibited by VR-HMD users include general discomfort, headache, vertigo, disorientation, pallor, fatigue, vomiting, nausea, stomach awareness, eye strain, difficulty focusing, blurred vision, increased salivation, and sweating $[8,9]$. Symptoms like these that appear following the VR experience can last for hours and, in some cases, even for a few days (9). Previous studies have reported visual and nonvisual symptoms associated with using VR-HMD. Han et al. compared the visual symptoms experienced by study participants after using two different displays and found that visual fatigue and visual discomfort were remarkably increased after watching a 3D movie using VR-HMD technology [10]. Another study conducted by Lee et al. compared eyestrain caused by $2 \mathrm{D}$ and $3 \mathrm{D}$ displays and reported the highest amount of eyestrain experienced by study participants was caused by 3D display use [11]. However, Turnbull et al. contended that the use of a VR headset for 40 minutes did not seem to affect the binocular vision status of their study participants as compared with when performing a real-world equivalent task [12].

The above findings clearly indicate that VR-HMD usage leads to visual symptoms and discomfort. Observed symptoms may indicate the occurrence of changes in some of the oculomotor functions (i.e., accommodation and/or vergence system parameters) at least for a short period of time. Some previous studies recorded changes in the accommodation and/or vergence systems of study participants after watching 3D movies using VR-HMDs [13, 14]. The present study sought to investigate the impact of playing 3D video games using VR-HMDs on the accommodation and vergence systems of healthy young adults. Also, this study sought to quantify the cybersickness symptoms experienced after using VR-HMD technology by employing the standardized Simulator Sickness Questionnaire (SSQ) [15].

\section{Material and Methods}

\subsection{Participants}

Study participants included both male and female students from the College of Applied Medical Science, King Saud University, Riyadh, Saudi Arabia. The study inclusion criterion was the presence of normal binocular vision, which was defined by certain ocular findings including no strabismus at distance or near using a cover test, at least 60 seconds of arc stereoacuity at near, a corrected distance visual acuity of $6 / 6$ in each eye, and no presbyopia. Subjects with any ocular and/or neurological diseases were excluded from this study to prevent the influence of selection biases. In total, 26 male and female young adults were able to complete this study with a mean \pm standard deviation age of $22.5 \pm 2$ years (range: 19-27 years). All participants were informed about the study and signed a written consent form before participating. This study followed the tenets of the Declaration of Helsinki and was approved by the King Saud University Office of Research Ethics.

\subsection{Measurements and Procedures}

This study consisted of six stages. First, different visual functions, including monocular visual acuity at distance assessed using the Snellen visual acuity chart, the Titmus stereoacuity test at near, and a cover test for determining the presence or absence of strabismus at both distance and near, were examined to determine whether participants met the criterion for inclusion (normal binocular vision) in this study. Then, participants were asked to fill out the SSQ to quantify baseline symptoms prior to using the VRHMD system. The SSQ is a standardized questionnaire designed to measure cybersickness symptoms that consists of three categories or subscales of symptoms, each of which measure a specific type of symptom. The first subscale of the SSQ is the ocular subscale $(\mathrm{O})$, which measures symptoms related to eyestrain and visual fatigue. The second subscale is the disorientation subscale (D), which measures symptoms related to balance and vestibular functions. Finally, the third subscale is the nausea subscale $(\mathrm{N})$, which measures symptoms such as nausea, salivation, burping, and vomiting. Each subscale consists of multiple items or questions; in this study, for each item, participants rated their baseline symptoms from zero points (none) to three points (severe). In addition to the three aforementioned subscales, the SSQ total score 
can be calculated to reflect the overall severity of cybersickness symptoms $[8,15]$.

Next, baseline clinical measurements of both the accommodation and vergence systems were collected. Clinical parameters linked to the accommodation system that were of interest in this study included the monocular amplitude of accommodation, assessed using the RAF pushup method; accommodation accuracy, assessed using the monocular estimated method; and negative relative accommodation (NRA), positive relative accommodation (PRA), and monocular and binocular accommodative facilities using $\pm 2.00 \mathrm{D}$ lens flippers. Meanwhile, clinical parameters linked to the vergence system that were of interest in this study included the amount of horizontal heterophoria at a distance and near, assessed using the Maddox rod test; the near point of convergence (NPC), assessed using accommodative targeting; the horizontal positive fusional vergence (PFV) and negative fusional vergence (NFV) ranges at both distance and near, assessed using step prisms (prism bar); and the vergence facility, assessed using (12 prism base-out, three prism base-in) prism flippers.

Fourth, all participants played two commercially available interactive 3D video games using the PlayStation VR-HMD gaming system from Sony (Tokyo, Japan). The first game was called ASTRO BOT Rescue Mission (SIE Japan Studio, Tokyo, Japan). In this game, players must control the movement of a robot by using their heads and bodies to move it forward and pass over obstacles at different distances, which requires different accommodative and vergence demands for success. Participants were asked to spend 20 minutes playing this game. The second game was Resident Evil 7: Biohazard (Capcom, Osaka, Japan), where players had to control the main character, who was searching for his wife, while solving puzzles and sometimes fighting other characters, which again placed different accommodative and vergence demands. Participants were asked to spend 25 minutes playing this game for a total of 45 minutes spent playing both video games together. The two video games were played in the same order by all study participants. The reasoning behind selecting these two games was to force participants to maintain a high level of activeness, motivation, and awareness throughout the whole playing session.

Finally, right after the video game session was over, the participants were asked to rate their symptoms by completing the SSQ again and clinical measurements of parameters relating to the accommodation and vergence systems were remeasured again to allow for differences between before and after the VR experience to be revealed. All measurements recorded after participants' VR gameplay were collected within 20 minutes of the end of their playing time.

\subsection{Statistical Analysis}

The normality of accommodative measurements, vergence measurements, and different SSQ scores was tested using the Shapiro-Wilk test. All of these variables were shown to be not normally distributed. Separately, the Wilcoxon signedrank test was used to compare the baseline measurements of median values of accommodative parameters, vergence parameters, and different SSQ scores with those recorded after VR gameplay. A p-value of less than 0.05 was considered to be statistically significant.

All of the clinical accommodative measurements that are normally measured monocularly were recorded for both the right and left eyes in all participants and included the amplitude of accommodation, accommodative accuracy, and monocular accommodative facility. The differences between measurements of the right and left eyes were not statically significant for all participants. For this reason, the study analysis was carried out using measurements of the right eye only from all participants.

With respect to the horizontal PFV and NFV ranges at both distance and near, the break-up points were considered for comparison between before and after VR gameplay. The analysis was carried out using the SPSS version 26 software program (IBM Corporation, Armonk, NY, USA)

\section{Results}

Mean, standard deviation, and median values for all accommodative and vergence measurements (before vs. after VR gameplay) are shown in Tables 1 and 2, respectively. Direct comparisons of different accommodative measurements based on median values showed that, among all accommodative measurements, the effect of playing VR video games was statistically significant for NRA, accommodative accuracy, and monocular and binocular accommodative facilities (Table 1). Other measurements did not present significant differences. At first, the PRA test result was observed to be approaching a significant effect level but ultimately did not reach this goal; however, even though the PRA did not reveal a statistically significant difference, the result appears clinically significant since the difference between before and after VR gameplay was 0.50 D (Table 1).

Table 1. Mean, standard deviation, median, and Wilcoxon signed-rank test values for all accommodative measurements before and after VR gameplay.

\begin{tabular}{lllc}
\hline Accommodation measurements & Mean (SD) & Median & W \\
\hline Amplitude of accommodation & & & -0.095 \\
(Before VR gameplay) & $12.08(3.51)$ & 12 & 0.924 \\
(After VR gameplay) & $11.58(4.11)$ & 10.5 & -2.038 \\
Negative relative accommodation & & & \\
(Before VR gameplay) & $2.68(0.79)$ & 2.5 & $0.042 *$ \\
(After VR gameplay) & $2.46(0.82)$ & 2.25 & \\
Positive relative accommodation & & & \\
\hline
\end{tabular}




\begin{tabular}{|c|c|c|c|c|}
\hline Accommodation measurements & Mean (SD) & Median & W & p-value \\
\hline (Before VR gameplay) & $2.35(1.44)$ & 2.25 & 1.953 & 0.051 \\
\hline (After VR gameplay) & $2.00(1.21)$ & 1.75 & & \\
\hline $\begin{array}{l}\text { Accommodative accuracy (monocular estimated method) } \\
\text { (Before VR gameplay) } \\
\text { (After VR gameplay) }\end{array}$ & $\begin{array}{l}-0.73(0.39) \\
-1.03(0.57)\end{array}$ & $\begin{array}{l}-0.75 \\
-1\end{array}$ & 2.790 & $0.005^{*}$ \\
\hline $\begin{array}{l}\text { Monocular accommodative facility } \\
\text { (Before VR gameplay) } \\
\text { (After VR gameplay) }\end{array}$ & $\begin{array}{l}10.62(4.29) \\
8.42(4.46)\end{array}$ & $\begin{array}{l}9.5 \\
7\end{array}$ & -4.006 & $<0.001^{*}$ \\
\hline $\begin{array}{l}\text { Binocular accommodative facility } \\
\text { (Before VR gameplay) } \\
\text { (After VR gameplay) }\end{array}$ & $\begin{array}{l}10.12(3.34) \\
8.35(3.89)\end{array}$ & $\begin{array}{l}11.5 \\
8.5\end{array}$ & -3.202 & $0.001 *$ \\
\hline
\end{tabular}

W: Wilcoxon signed-rank test value

SD: standard deviation; VR: virtual reality

(*): The difference is statically significant at the 0.05 level

With respect to vergence measurements, the majority of direct comparisons, based on an analysis of median values, did not indicate a significant difference existed between before and after playing VR video games (Table 2); ultimately, the PFV amplitude at near, NFV amplitude at near, and vergence facility were the only parameters that showed significant differences between sessions (Table 2).

Table 2. Mean, standard deviation, median, and Wilcoxon signed-rank test values for all vergence measurements before and after VR gameplay.

\begin{tabular}{|c|c|c|c|c|}
\hline Vergence measurements & Mean (SD) & Median & W & p-value \\
\hline $\begin{array}{l}\text { Phoria at distance } \\
\text { (Before VR gameplay) }\end{array}$ & $2.19(3.72)$ & 2.50 & -0.661 & 0.509 \\
\hline (After VR gameplay) & $2.27(3.05)$ & 2 & & \\
\hline $\begin{array}{l}\text { Phoria at near } \\
\text { (Before VR gameplay) } \\
\text { (After VR gameplay) }\end{array}$ & $\begin{array}{l}0.35(4.5) \\
0.00(4.20)\end{array}$ & $\begin{array}{l}-1.00 \\
-0.50\end{array}$ & -0.582 & 0.561 \\
\hline PFV at distance & & & & \\
\hline $\begin{array}{l}\text { (Before VR gameplay) } \\
\text { (After VR gameplay) }\end{array}$ & $\begin{array}{l}17.19(4.8) \\
16.46(5.1)\end{array}$ & $\begin{array}{l}16 \\
16\end{array}$ & -0.720 & 0.471 \\
\hline NFV at distance & & & & \\
\hline $\begin{array}{l}\text { (Before VR gameplay) } \\
\text { (After VR gameplay) }\end{array}$ & $\begin{array}{l}10.42(5.7) \\
9.54(3.5)\end{array}$ & $\begin{array}{l}9 \\
8\end{array}$ & -0.342 & 0.732 \\
\hline $\mathrm{PFV}$ at near & & & & \\
\hline $\begin{array}{l}\text { (Before VR gameplay) } \\
\text { (After VR gameplay) }\end{array}$ & $\begin{array}{l}21.77(9.4) \\
17.65(8.1)\end{array}$ & $\begin{array}{l}18 \\
15\end{array}$ & -3.105 & $0.002 *$ \\
\hline NFV at near & & & & \\
\hline (Before VR gameplay) & $15.42(4.6)$ & 14 & -3.105 & $0.002 *$ \\
\hline $\begin{array}{l}\text { (After VR gameplay) } \\
\text { NPC }\end{array}$ & $12.92(3.7)$ & 12 & & \\
\hline $\begin{array}{l}\text { (Before VR gameplay) } \\
\text { (After VR gameplay) }\end{array}$ & $\begin{array}{l}5.46(1.7) \\
5.88(2.0)\end{array}$ & $\begin{array}{l}5 \\
6\end{array}$ & 1.491 & 0.136 \\
\hline $\begin{array}{l}\text { Vergence facility } \\
\text { (Before VR gameplay) } \\
\text { (After VR gameplay) }\end{array}$ & $\begin{array}{l}10.65(2.6) \\
8.98(3.8)\end{array}$ & $\begin{array}{l}11 \\
9\end{array}$ & -2.668 & $0.008^{*}$ \\
\hline
\end{tabular}

W: Wilcoxon signed-rank test value

NFV: negative fusional vergence; NPC: near point of convergence; PFV: positive fusional vergence; SD: standard deviation; VR: virtual reality and NPC:

Near Point of Convergence.

$(*)$ : The difference is statistically significant at the 0.05 level

Table 3 shows means, standard deviations, and medians for different subscales and total score of the SSQ. Direct comparisons, conducted using the Wilcoxon signed-rank test, between before and after VR gameplay were statistically significant for both the total score and all subscale scores of the SSQ. The largest alterations in cybersickness symptoms after playing VR games pertained to the nausea and oculomotor subscales.

Table 3. Mean, standard deviation, median, and Wilcoxon signed-rank test values for subscale and total scores of SSQ before and after VR gameplay.

\begin{tabular}{|c|c|c|c|c|}
\hline SSQ & Mean (SD) & Median & W & p-value \\
\hline Nausea subscale & & & & \\
\hline $\begin{array}{l}\text { (Before VR gameplay) } \\
\text { (After VR gameplay) }\end{array}$ & $\begin{array}{l}4.40(6.2) \\
37.1(33.7)\end{array}$ & $\begin{array}{l}0 \\
28.62\end{array}$ & 3.893 & $<0.001$ \\
\hline Oculomotor subscale & & & & \\
\hline (Before VR gameplay) & $8.45(13.1)$ & 0 & 3.953 & $<0.001$ \\
\hline
\end{tabular}




\begin{tabular}{|c|c|c|c|c|}
\hline SSQ & Mean (SD) & Median & $\mathbf{W}$ & p-value \\
\hline (After VR gameplay) & $39.6(30.5)$ & 34.11 & \multirow{5}{*}{3.438} & \multirow{5}{*}{0.001} \\
\hline Disorientation subscale & & & & \\
\hline (Before VR gameplay) & $5.35(9.7)$ & 0 & & \\
\hline (After VR gameplay) & $36.4(47.1)$ & 20.88 & & \\
\hline Total score & & & & \\
\hline (Before VR gameplay) & $35.8(145.6)$ & 3.74 & \multirow[t]{2}{*}{3.419} & \multirow{2}{*}{0.001} \\
\hline (After VR gameplay) & $44.4(37.3)$ & 37.40 & & \\
\hline
\end{tabular}

W: Wilcoxon signed-rank test value

SD: standard deviation; VR: virtual reality

\section{Discussion}

Playing 3D video games using a VR headset is gaining significant popularity among younger generations; thus, investigating whether VR-HMD technology is dangerous to our visual system is very important. The present study aimed primarily to understand how playing 3D video games using VR-HMDs affects oculomotor functions, at least for a short period of time. The oculomotor functions that were investigated in this study included accommodation and vergence parameters that are routinely assessed in optometry and ophthalmology clinics. Meanwhile, this study also sought to subjectively evaluate the symptoms of VR-HMD users with the SSQ.

This study revealed that playing $3 \mathrm{D}$ video games for 45 minutes using VR-HMD technology had significant effects on the majority of clinical accommodation measurements. The study findings included a significant reduction in the NRA test results following VR gameplay, which means that a significant reduction in the ability of the eye to maintain a clear and single image while the accommodation is relaxed occurred among the study participants. Similarly, the study findings included a reduction in the PRA test results, which means there was a reduction in the ability of the eye to maintain clear and single image while the accommodation is stimulated among the study participants; however, this effect did not reach a statistically significant level. Overall, accommodative accuracy was shown to be significantly increased after VR gameplay, which means that our study participants' eyes tended to overaccommodate after their respective VR gameplay. Finally, the values of both the monocular and binocular accommodative facilities were reduced after VR gameplay, which means a significant reduction in the ability of the eyes of our study participants to make rapid and accurate accommodative changes, occurred during the course of this investigation. Meanwhile, the amplitude of accommodation was not altered at all after VR gameplay.

Changes induced by playing 3D video games using VR-MHD technology were reflected in differences in the NRA and accommodative accuracy values, representing typical diagnostic signs of "accommodative excess," a phenomenon that arises when the accommodation system improperly overaccommodates for the stimuli. Separately, reductions in the monocular and binocular accommodative facilities constitute typical diagnostic signs of "accommodative infacility." Meanwhile, "accommodative insufficiency" is associated with reductions in the amplitude of accommodation and PRA. This could be the reason for why both did not show statistically significant differences between before and after VR gameplay [16].

The results of this study are in agreement with a previous investigation that found the amplitude of accommodation did not change after VR gameplay [13]. In addition, previous studies have also revealed findings of overaccommodation like as seen in this study. Ha et al. observed an increase in the amount of myopic refractive error in the majority of their study participants right after using VR-HMDs. Another study in support of this conclusion found that unaided visual acuity at near was significantly reduced after viewing 3D imagery using HMDs, which may reflect overaccommodation activity [14]. Elsewhere, other research found that viewing a VR movie using VR-HMD technology can significantly increase the thickness of the choroid, which resulted in greater myopic defocus among the users [12].

It is expected that accommodative lead and myopic shift (i.e., too much accommodation) will be prevalent among video game players using VR-HMD technology because VR users have to converge their eyes when looking through the lenses of the device. As eyes look off the lens centers, a baseout prismatic effect is induced, which results in an increased demand of accommodative-convergence [17]. Regardless of these results, it has been reported that myopic shift can be induced after using some instruments such as microscopes, which is known as "instrument myopia" [18].

The results of our study showed that exposure to VR headsets appear to have some effects on the vergence system. Both PFV and NFV amplitudes at near were significantly decreased after VR gameplay. It should be noted that low NFV at near is considered a diagnostic sign for "accommodation excess," which supports our accommodation measurement findings. In addition, the vergence facility was another aspect of the vergence system that was affected following VR gameplay. This suggests that VR game players are less able than those who do not play VR games to make rapid and accurate vergence changes, which is consistent with the analysis of accommodative facility in this study. It seems that, since playing $3 \mathrm{D}$ video games using VR-HMDs requires users to change their accommodative and vergence demands continuously, these individuals become tired over time and their abilities are affected. Other vergence measurements collected in this 
study did not significantly change after VR gameplay, including the amount of heterophoria at distance and near, PVF and NFV at distance, and the NPC.

Results from previous studies revealed no significant effects of using 3D VR-HMDs on the amount of heterophoria at distance or near or concerning fixation disparity, which is consistent with the findings of this study [13, 14, 19]. The amount of heterophoria in most studies appears not to have been affected after using VR-HMD for 30 minutes. However, a significant esophoric shift at distance (i.e., eyes tend to converge) was reported after 80 minutes of VR gameplay [14] The esophoric effect that was observed by Kozulin et al. supports the results of this study where VR users tended to experience accommodation excess given that esophoria is another reflection of overaccommodation activity [14]. A recently published study found that both the accommodative and vergence facilities reduced significantly after playing with the VR headset, which support the results of this study [20].

Increased interest in playing 3D video games using VRHMD systems likely accounts for the rise in cybersickness symptoms. The majority of participants of this study reported significant increments in all types of symptoms measured by the SSQ, including nausea, oculomotor, and disorientationrelated symptoms. In addition, the total score of the SSQ was also significantly increased for the majority of participants. Among the different types of symptoms, the most significant changes observed after VR gameplay were linked to nausea and oculomotor symptoms.

It is already known from previous studies that watching 3D stereoscopic movies or video games without using a VR headset can still lead to both visual and nonvisual symptoms. Importantly, these symptoms may reflect certain oculomotor function changes [7, 21]. Stone found that cybersickness symptoms were quite limited when $3 \mathrm{D}$ interactive video games played using VR headsets were combined with physical motion for about 20 minutes [8]. Han et al. reported that $3 \mathrm{D}$ video games using VR headsets induced more cybersickness symptoms when compared with video games played using smartphones [10]. One study found that watching 3D video content using a VR headset for 80 minutes caused more visual and nonvisual symptoms as compared with using the headset for 30 minutes in some subjects [14].

Nevertheless, the findings of this study have some limitations that should be discussed. First, the study sample size was considerably small. Second, young adults were the only age group included. Third, the duration of viewing was relatively short. Consequentially, the present findings may not accurately demonstrate the oculomotor effects experienced by the majority of VR-HMD users, especially those who spend a long time using this technology. Thus, future studies should investigate the effects of playing interactive 3D video games using VR-HMDs over a longer duration. A study performed by Pölönen et al. determined that there were no significant changes in the amount of heterophoria and the amplitude of accommodation after two hours of playing $3 \mathrm{D}$ video games on video screens among a sample of young children [22]

\section{Conclusion}

In conclusion, results from this study showed that playing interactive 3D video games using VR-HMDs for 45 minutes undoubtedly induced some clinical oculomotor changes. The most obvious clinical oculomotor changes were accommodative excess, accommodative infacility, limited fusional vergence amplitude at near, and vergence infacility. In addition, most of the study participants subjectively reported increments in visual and nonvisual symptoms. Given these findings, 3D video game players are encouraged to check their eyes on a routine basis, especially their binocular vision functions, to prevent or minimize any cybersickness symptoms or oculomotor changes that may occur after playing video games.

\section{Acknowledgements}

The authors would like to extend their sincere appreciation to the Deanship of Scientific Research and the Research Center at the College of Applied Medical Sciences at King Saud University for funding this work.

The authors thank the Deanship of Scientific Research and RSSU at King Saud University for their technical support.

The authors declare that there is no conflict of interest or any financial disclosure that could interfere with the results of this study.

\section{References}

[1] Anthes C, García-Hernández RJ, Wiedemann M, Kranzlmüller D. State of the art of virtual reality technology. In: IEEE Aerospace Conference Proceedings. 2016.

[2] Tam WJ, Speranza F, Yano S, Shimono K, Ono H. Stereoscopic 3D-TV: Visual comfort. IEEE Trans Broadcast. 2011; 57: 3335-346.

[3] Howarth PA, Costello PJ. The occurrence of virtual simulation sickness symptoms when an HMD was used as a personal viewing system. Displays. 1997; 18: 107-116.

[4] Rebenitsch L, Owen C. Review on cybersickness in applications and visual displays. Virtual Real. 2016; 20: 101125.

[5] Scheiman M, Wick B. Clinical management of binocular vision: Heterophoric, accommodative, and eye movement disorders: Fourth edition. Clinical Management of Binocular Vision: Heterophoric, Accommodative, and Eye Movement Disorders: Fourth Edition. 2013.

[6] Lambooij M, Ijsselsteijn W, Fortuin M, Heynderickx I. Visual discomfort and visual fatigue of stereoscopic displays: A Review. Journal of Imaging Science and Technology. 2009; 53: 30201-1.

[7] Zeri F, Livi S. Visual discomfort while watching stereoscopic three-dimensional movies at the cinema. Ophthalmic Physiol Opt. 2015; 35: 271-282. 
[8] Stone III WB. Psychometric evaluation of the Simulator Sickness Questionnaire as a measure of cybersickness. 2017; 143.

[9] LaViola JJ. A discussion of cybersickness in virtual environments. ACM SIGCHI Bull. 2000; 32: 47-56.

[10] Han J, Bae SH, Suk H-J. Visual Discomfort and Visual Fatigue: Comparing Head-Mounted Display and Smartphones. J Ergon Soc Korea. 2017; 36: 293-303.

[11] Lee EC, Heo H, Park KR. The comparative measurements of eyestrain caused by 2D and 3D displays. IEEE Trans Consum Electron. 2010; 56: 1677-83.

[12] Turnbull P., Phillips J. Ocular effects of virtual reality headset wear in young adults. Sci Rep. 2017; 7: 1-9.

[13] Ha SG, Na KH, Kweon IJ, Suh YW, Kim SH. Effects of headmounted display on the oculomotor system and refractive error in normal adolescents. J Pediatr Ophthalmol Strabismus. 2016; 53: 238-245.

[14] Kozulin P, Ames SL, McBrien NA. Effects of a head-mounted display on the oculomotor system of children. Optom Vis Sci. 2009; 86: 845-856.

[15] Kennedy RS, Lane NE, Berbaum KS, Lilienthal MG. Simulator Sickness Questionnaire: An Enhanced Method for Quantifying Simulator Sickness. Int J Aviat Psychol. 1993; 3: 203-20.
[16] American Optometric Association. Optometric Clinical Practice Guideline: Care of the pation with Accommodative and Vergence Dysfunction. Am Optom Assoc. 2011.

[17] Schor C. The influence of interactions between accommodation and convergence on the lag of accommodation. Ophthalmic Physiol Opt. 1999; 19: 134-150.

[18] Leibowitz HW, Owens DA. Anomalous myopias and the intermediate dark focus of accommodation. Science. 1975; 189: 646-648.

[19] Tychsen L, Foeller P. Effects of Immersive Virtual Reality Headset Viewing on Young Children: Visuomotor Function, Postural Stability, and Motion Sickness. Am J Ophthalmol. 2020; 209: 151-159.

[20] Munsamy AJ, Paruk H, Gopichunder B, Luggya A, Majola T, Khulu S. The effect of gaming on accommodative and vergence facilities after exposure to virtual reality headmounted display. J Optom. 2020.

[21] Zhang L, Zhang YQ, Zhang JS, Xu L, Jonas JB. Visual fatigue and discomfort after stereoscopic display viewing. Acta Ophthalmol. 2013; 91: 149-153.

[22] Pölönen M, Järvenpää T, Bilcu B. Stereoscopic 3D entertainment and its effect on viewing comfort: Comparison of children and adults. Appl Ergon. 2013; 44: 151-160. 